\title{
Brain Evolution: Brainstem and Cerebellum followed by Spinal Cord
}

\author{
Komi Assogba* \\ Department Neurology, Togo
}

*Corresponding author: Assogba K, Professor of Neurology, Campus Teaching Hospital of Lomé, Togo.

Received Date: November 23, 2018

Published Date: December 04, 2018

\section{Opinion}

The brain is a complex organ that serves as the center of the nervous system. The brain exists in human, animals and trees, from microscopic size in viruses to the complex composition in human being. The brain is composed of the cerebrum, the brainstem and the cerebellum and followed by the spinal cord. This very specific organ is located inside the skull bones of the head. The shape and size of the brain varies greatly between species from more primitive ones to advanced brains.

The purpose of this opinion is to focus on human brain and its disorders with exclusion of the spinal cord. The brain is the most protected organ. The cerebrum is divided into two cerebral hemispheres. Each hemisphere is conventionally divided into five lobes; named the frontal, temporal, parietal, insular and occipital lobes. A lobe is divides in circumvolution with small specific areas call aires. The left and right hemispheres are broadly similar anatomically but different in function such as language in the left and visual-spatial ability in the right. The two hemispheres are connected by commissural nerve tracts to share on time the same information and execute the body function together.

The human brain contains approximately 70 billion neurons. Each neuron is constituted by body cellar and axon that connected by synapses to several thousand other neurons. The neuron or nerve cell is the functional unit of the brain. The nerve cell is the first electrical and excitable cell of the body. This electrical power is the base of specific signal pulse that helps neuron to be connected one to another from and to the brain in the entire body, to execute the human willing and desire.

The function of the brain is lead by the neuron to exertcentralized control over the other organs of the body. The neuron acts by controlling other body cells activities from mitosis to hormone secretion, excretion and clean wastes from the body and reinforce human defense system. The brain is the heart that centralized all functions by generating patterns of activity, integrating capabilities, and coordinated input and output of responses to adapt human behavior to the environment.

Some basic types of responsiveness can be mediated by the spinal cord or peripheral ganglia, but sophisticated purposeful control of behavior based on complex sensory input and output requires the information integrating capabilities of a centralized brain. The brain functions concern movement control, sensory responsiveness, intelligence, memory, thought, and sleeping. Considerable details of neuron operating system are now well understood but great remain to be solved regarding some specific functions such as fetal development to birth, cognitive, behavior, environment adaptation and saving life.

The neuron function, excitability and well being is depending on multiple factors among which we can list but not exhaustive, the nerve cell development and constitutional, brain protective system, blood circulation in arteries and veins, cerebrospinal fluid circulation, hormones and other secretion, other organ and system including immunity defense system.

During centuries, there were evolution of sciences and history of neurosciences. From the ancient Egyptian practices to $21^{\text {st }}$ century, via Roman Empire and the renaissance period, there is evidence of neuroscience practice throughout periods of history. All these research period had conducted to identify the specialization of specific brain areas functions and neural plasticity by Paul Broca, Carl Wernicke and Korbinian Brodmann. With little known about nerve cell function in detail, great and big challenges and myths remain yet to be solved regarding the brain disorders. As much complex as the brain functions are, as difficult, multiple and varied are the brain disorders. The disorder occurs when there is damage or disruption to the brain.

There are many risk factors leading to brain damage. These factors are developmental errors, birth period disturbances, polluted environmental, bad lifestyle, stressful life experiences, 
alcohol and drugs misusing, epidemic, natural disasters, and other living destructive conditions. Everyone at any time and any age can develop brain disorder by exposure to these risk factors. A brain disorder is all range of disabilities that affect the brain function including physical, memory, personality and intellectual aspects from antenatal period to oldest people. These disorders include traumatic brain injury, stroke and other vascular diseases, hypoxic and electrolytes imbalances from internal organs dysfunction, Parkinson's and Alzheimer's disease and other degenerative diseases, alcohol and other drugs consumption, brain tumors, epilepsy, brain infections and inflammatory diseases, and brain maturation dysfunction. Long-term effects will be different for each person and will also vary depending on the type of brain disorder. Some disorders, degenerative and inflammatory, will leave the cognition intact, but have dramatic impacts on the body's ability to control movement, while vascular and infectious damages will give disabilities and cognitive impairment. Physical signs and symptoms can vary widely between the disorders, range from the simplest common ones, including headaches and chronic pain, movement disorders and paralysis, loss of sensory all type, dizziness and balance problems, seizures, eyesight and hearing problems, and loss of behavioral and mood control, to brain dementia. Some brain disorders appearance mechanism is well understood through many sciences and neurosciences studies, however a lot of them still unidentified.

The diagnostic of brain disorders is made on the base of clinical signs and symptoms help by medical imaging technologies. These techniques were developed and increase progressively in performance from the beginning of earth life up to date. The brain exploration useful methods are composed of human circulation balance, microscope and biology, structural radiography, electroencephalography and evocated potential myographic recordings, computerized axial tomography and magnetic resonance imaging, SPECT and PET scans with radioactive neuroimaging, and functional MRI. The outcome for people with brain disorders depends on the type and severity of the brain damage. Some conditions are easily treated with medication and therapy, and millions of peoples with brain disorders live perfectly normal live. Other disorders, like vascular and neurodegenerative diseases, personality and behavioral, and some traumatic brain injuries, have no cure. People with these conditions often face permanent changes in their mental and behavior balance, and motor and sensory abilities. In these cases, treatment will try to help manage the illness to get independence as much as possible.

Medicine drugs are made by using neurotransmitters, inhibitors, stimulators, psychoactive, or general drugs mechanisms. Despite many available technical therapies such as surgery, deep brain stimulation, stem cell therapy, spiritual and traditional therapy, rehabilitation, physiotherapy, psychotherapy, and speech therapy, many brain disorders remain untreatable. To date lot of new drugs are in trial essay to fill this gap, as the brain areas and the brain disorders are remaining mysterious.

Many brain disorders may be complicated in different types and some of them will end by death. The diagnosis of brain death is often required to be highly rigorous, to be certain that the condition is irreversible. Legal criteria vary, but in general, require neurological examinations by two independent physicians. The exams must show complete and irreversible absence of brain function and may include two flat-line EEGs 24 hours apart.

Traditionally, both the legal and medical communities determined death through the permanent end of certain body functions, especially respiration and heartbeat. With the increasing ability of the medical community to resuscitate people with no respiration and heartbeat, or other external signs of life, the need for another definition of death occurred, raising questions of legal death. This gained greater urgency with the widespread use of life support equipment, as well as rising capabilities and demand for organ transplantation. Despite the medical high technologic therapy available, there are no yet any transplantation brain programs, and a few preventive measures for brain disorders are known.

The disorders effects can be temporary or permanent, and range from mild injury, to a very severe damage that may cause prolonged loss of consciousness. Unfortunately, whatever we do and whenever, the decease will come by the brain natural end evolution itself or many disorders that will end the brain function. There are many challenges which need to overcome to fill the gaps regarding the brain and brain disorders. Numerous areas brain functions remain undiscovered. The researches in neuroscience must focus in the coming century years on the brain disorders therapy to high improve life expectancy and lifetime. The science of the brain will be the head of the medical sciences and the neurosciences, the medical sciences leader for the future.

Almost everything we do, say and think is controlled by our brain, so when our brain is injured or sick it has the potential to affect every aspect of life. A brain disorder can affect anyone at any age. The personal risk depends on the genetics, and the exposure to environmental risk factors and lifestyle. Normally, human life must be ended by natural degenerative death, after thousand years. Human being has to follow best lifestyle practice, safe birth, safe and clean environment, to obtain good functional brain to avoid and prevent any brain damage and diseases. Neural physician, medical caregivers and the whole population have to play their partition in the brain good care and brain disorders management.

\section{Acknowledgement}

None.

\section{Conflict of Interest}

No conflict of interest. 